\title{
Sentinel Seals, Safe Cats, and Better Treatments
}

\subsection{A Review}

This chapter examines some additional implications of the Toxoplasma gondii problem. Especially impressive is evidence suggesting that this parasite is contaminating our land and waterways. The infection of marine mammals by this parasite is little known but disturbing. The chapter then proposes various solutions to the problem: specifically by better control of feral cats to decrease the distribution of $T$. gondii oocysts; by research, including the development of vaccines for cats; by improving the treatment of toxoplasmosis in humans; and by educating the public regarding these issues.

We began this story by establishing two preliminary facts. First, many human diseases are caused by infectious agents transmitted to us from animals, the Covid pandemic being the most recent example. Second, there is abundant evidence that infectious agents can cause psychosis. We then examined in detail Toxoplasma gon$\mathrm{dii}$, a protozoan parasite carried by cats. It is known to infect 40 million Americans, most of them apparently being asymptomatic. However, it has been clearly established that this parasite causes congenital problems of the central nervous system, estimated to be approximately 3800 cases per year in the United States, and is also a major cause of eye diseases, estimated to be approximately 4800 symptomatic cases per year. In addition, this parasite is a risk factor for causing cerebral toxoplasmosis in individuals who are immunosuppressed, including patients with HIVAIDS, individuals receiving organ transplants, and cancer patients being treated with immunosuppressive drugs. Toxoplasmosis causes an estimated 71 deaths each year in the United States, mostly due to cerebral toxoplasmosis.

We then examined in detail the evidence linking Toxoplasma gondii to the cause of some cases of psychosis. Four facts are supportive of such a link. First, Toxoplasma gondii is known to cause psychotic symptoms. Next, among individuals with schizophrenia, those who are infected with this parasite have been shown to have more severe symptoms. Third, many studies have shown that individuals with psychosis, compared to controls, are significantly more likely to have antibodies against 
this parasite, indicating a past infection. Finally, some but not all studies have reported that adults with psychosis, compared to controls, were significantly more likely as children to have lived in a house with cats.

To further explore a possible link between Toxoplasma gondii and psychosis, we next looked at the unusual 800-year history of cats which transitioned from being perceived as agents of Satan to becoming cherished pets. We found that there appears to be a correlation between the historic rise of cats as pets and the increasing incidence of psychosis. Finally, we examined several epidemiological aspects of schizophrenia, such as the fact that it is more common in cold climates, to ascertain whether these aspects can be explained by infection with Toxoplasma gondii. Overall, the evidence linking this parasite to the cause of some case of psychosis is strong. One estimate of the number of cases of psychosis that might be linked to Toxoplasma gondii suggested about $20 \%$, or 15,000 new cases of psychosis each year in the United States.

In addition to examining the possible link between Toxoplasma gondii and psychosis, we also briefly reviewed studies that have attempted to link this parasite to other diseases and conditions. The strongest evidence for a $T$. gondii link from studies done to date is for some cases of brain cancer, epilepsy, and rheumatoid arthritis. In addition, a small number of motor vehicle accidents may be linked to $T$. gondii based on the fact that the parasite slows human reaction times.

In summary, the importance of this relatively unknown parasite has been greatly underestimated, especially given the fact that it is known to infect approximately 40 million Americans. The next step is to ascertain the magnitude of the toxoplasmosis problem.

\subsection{What Is the Magnitude of the Problem?}

Since cats are the definitive host for $T$. gondii, solutions to the problems caused by this parasite will inevitably involve them. As noted in Chap. 1, the United States is thought to have approximately 90 million owned cats and 50 million feral cats for a total of 140 million. Studies have reported that the fecal production of a cat is approximately 40 grams per day. Thus the total daily fecal production for all the cats in the United States would be approximately 5.6 billion grams. Converted into pounds this would be 12,334,800 million pounds or 6167 tons. To visualize this, imagine a train with 62 standard boxcars, each of which carries 100 tons of freight. Then imagine the freight train being two thirds of a mile in length; this is what would be required to carry 1 days' fecal output for all the cats in the United States $[1,2]$.

Studies have shown that at any given time approximately $1 \%$ of all cats are excreting oocysts. One percent of 5.6 billion grams is 56 million grams of oocystinfected cat feces which would be produced each day in the United States. Each gram of infected cat feces may contain between 1 million and 13 million oocysts; assuming a mean of 6 million oocysts per gram for the 56 million grams of infected cat feces would produce a total of 336 trillion $(336,000,000,000) T$. gondii oocysts 
each day. Although it is not known how many oocysts are needed to infect a human, studies have been done in pigs which weigh about the same as humans, and it only took one oocyst to infect them [3,4].

One of the most striking aspects of $T$. gondii oocysts is their viability. They are remarkably hearty, especially if they are deposited in shady, moist, and temperate conditions. In Texas, under outdoor shaded conditions with a mean air temperature of $19.5^{\circ} \mathrm{C}$, oocysts remained viable during a 13-month experiment. In Kansas, oocysts were buried in loose soil and remained viable for 18 months. Oocysts maintained experimentally at $4{ }^{\circ} \mathrm{C}$ in seawater or freshwater remained viable for 24 and 54 months, respectively. Oocysts also survived for over a year in vials of $2 \%$ sulfuric acid at $4{ }^{\circ} \mathrm{C}$. Because almost all of these studies were terminated while at least some of the oocysts were still viable, we really do not yet know the outer limit of viability for T. gondii oocysts deposited under various environmental conditions. What we do know is that many oocysts are infective for a long time [5-9].

\subsection{Oocyst Contamination of Soil and Water}

Given the fact that cats in the United States produce 336 trillion T. gondii oocysts each day and that some of these oocysts may survive for 2 years or longer, it is important to consider the fate of these oocysts. In urban and suburban areas, the majority would be deposited in loose soil in gardens or in public or private children's play areas or sandboxes. In rural areas the oocysts might be deposited in feed piles in the barn or in loose soil in the field, there to be ingested by grazing animals such as cows and sheep. In recent years many studies have been carried out to assess the degree of contamination of soil and water by the oocysts. Because the various studies used different methods of sampling and measurements of T. gondii's presence, the results of the studies cannot be usefully compared with each other.

In California in Santa Cruz and Santa Clara Counties, six public parks and a community garden were sampled for $T$. gondii oocysts during the spring, summer, and fall seasons. Feral cat colonies were known to frequent all seven sites which were heavily used for public recreation. Three of the seven sites had positive samples for T. gondii, all in the fall season. At the Santa Cruz State Park, 20 of 27 samples were positive. Another study in California was carried out in three communities in the Morro Bay region. Among 12,244 households, researchers identified 7284 owned cats and an additional 2046 feral cats. Based on the information regarding cat defecation and cat litter disposition in these communities, the researchers calculated that the oocyst burden for the land area of residential housing ranged between 9 and 434 oocysts per square foot of land area [10-12].

Studies from several other countries clearly confirm that soil contamination by T. gondii oocysts is now worldwide. A study similar to the Morro Bay study was carried out in three communities in rural France and reported a similar oocyst burden of between 3 and 335 oocysts per square foot. In Brazil, T. gondii oocysts were isolated from ten soil samples taken from the playgrounds of 31 elementary schools; the authors suggested that these results indicated a wide distribution of $T$. gondii 
oocysts around elementary schools in the region. In a village in Panama, it was estimated that the oocyst burden in soil near houses where cats were regularly fed varied from 18 to 72 per square foot. In Poland, T. gondii oocysts were isolated from 18 of 101 soil samples taken from places thought to be favored by cats for defecation: sandboxes, playgrounds, parks, gardens, and areas around rubbish dumps. In Iran T. gondii DNA was identified in 18 of 200 soil samples taken from public parks, playgrounds, sand pits, and around rubbish dumps. In Pakistan T. gondii DNA was found in 42 of 250 soil samples taken from parks, playgrounds, and sandpits where children play. In China T. gondii was found in 58 of 252 soil samples taken from 6 public parks in Wuhan. Finally, a recent study from 6 provinces in China reported the results of soil sampling from 420 sites at schools, parks, farms, and coastal beaches. Out of the 420 sites, 136 (32\%) were positive for T. gondii DNA. The evidence of high soil contamination in the Chinese samples is especially concerning since keeping pet cats under Chairman Mao was discouraged and has only become common in the last two decades. A recent review of 22 such studies concluded: "The results are a cause for concern because various areas studied across the world are rather highly contaminated with toxoplasma oocysts" [13-22].

The loose sand in children's sandboxes/sandpits are especially attractive to cats as places to defecate. A study in Japan quantified the frequency of cat defecations in three public sandboxes by using night lights and a camcorder. Over almost 5 months, they recorded 961 cat defecations in the sandboxes. Assuming that $1 \%$ of the cats were shedding oocysts when they defecated and that the oocysts remained viable for at least 18 months, it is possible that the oocyst contamination in such a sandbox could reach over 1 million oocysts per square foot. Given the frequency with which young children put their fingers in their mouth, the chances of becoming infected with toxoplasmosis while playing in such a sandbox would be very high [23, 24].

However, it is not only soil samples that are being contaminated by $T$. gondii oocysts. Streams, rivers, and even the ocean are becoming contaminated to a remarkable degree. This occurs when cat feces are carried by rainwater into waterways and also when cat litter is flushed down the toilet. Increasingly, this is resulting in oocyst contamination of drinking water. In 1995, for example, a drinking water reservoir on Vancouver Island became contaminated with $T$. gondii oocysts resulting in at least 2984 human infections of which 100 had symptomatic toxoplasmosis. Contamination of drinking water reservoirs by $T$. gondii has also led to several outbreaks of toxoplasmosis in Brazil, including 1 involving 290 cases and another involving 155 cases $[25,26]$.

The consequences of the runoff of infected cat feces into the ocean have become abundantly clear. Between 1998 and 2004 along the California coast, 52\% of the dead and $38 \%$ of the live sea otters tested were infected with T. gondii. One of the consequences of such infections is that sea otters infected with $T$. gondii have been shown to be almost four times more likely to be attacked by sharks compared to sea otters not infected. It is thought that the T. gondii in the cat feces are taken up by mussels and other shellfish which are then eaten by the sea mammals. And it is not just sea otters. Between 2004 and 2009, 161 mostly stranded sea mammals on the coast of Oregon, Washington, and southern British Columbia were examined. They 
included 95 seals, 37 porpoises, 19 sea lions, 5 sea otters, 4 whales, and 1 dolphin. Ninety-four of the 161 , or $58 \%$, were found to be infected with $T$. gondii. On the other side of the North American continent between 2009 and 2012 in the estuary of the St. Lawrence River, 34 beached beluga whales were tested. Fifteen of the 34, or $44 \%$, were found to be infected with $T$. gondii. It was hypothesized that the source of the infection was cat feces from the estimated 2 million cats in Quebec, especially from the downriver urban Montreal area. T. gondii was also said to be responsible for the death of 406 seals in 2012 off the coast of Nova Scotia [27-31].

A summary of the studies published in 2020 makes abundantly clear how extensively toxoplasmosis has infected marine mammals. Based on antibody studies, at least nine marine mammal species have been proven to be infected with $T$. gondii: seals, sea otters, dolphins, porpoises, manatees, sea lions, whales, walruses, and dugong. Based on autopsy reports, toxoplasmosis has been proven to be the definitive or a contributory cause of death in some mammals in all of these species except the last two. For example, in southern sea otters, an endangered species, T. gondii was reported to be the primary cause of death for $17 \%$ and a contributory factor for an additional $12 \%$ based on sea otter deaths examined histologically between 1998 and 2001. And the effect is worldwide. Infected dolphins, for example, have been found off the coasts of the United States, Canada, Mexico, England, Spain, Italy, Russia, and the Philippines [32].

The contamination of the ocean by $T$. gondii from cat feces off the coasts of highly populated areas is alarming enough, but the situation is even worse. In 2014 it was announced that a beluga whale taken by Inuit hunters in the Beaufort Sea north of Alaska had tested positive for T. gondii. In recent decades cats have become popular pets in Eskimo communities. And in Antarctica 52\% of Weddell seals and $23 \%$ of elephant seals have been found to be infected with T. gondii. Since the seals migrate, it is unknown where they became infected; one possibility is Macquarie Island which lies halfway between Antarctica and New Zealand. For many years the island was overrun with feral cats, the consequences of their introduction in the early nineteenth century by seal harvesters. As one recent paper concluded, "It is evident from the review that contamination of marine aquatic life with $T$. gondii is widespread and we are surrounded by a sea of toxoplasma" [33-35].

\subsection{Solutions to the Problem}

Given what we know and, more important, what we don't know about toxoplasmosis, it does not seem wise to be contaminating our land and water with infective oocysts. As a veterinary problem, we know that all warm-blooded animals can be infected and that it is a major cause of fetal loss in sheep and goats. As a human problem, we know that 40 million Americans are infected and that the infective oocysts are a significant cause of congenital brain problems, eye disease, and encephalitis for individuals who are immunocompromised due to HIV-AIDS, organ transplantation, or cancer treatment. Additional evidence suggests that toxoplasma may well play a role in causing some cases of psychosis as well as possibly causing 
some cases of brain cancer, epilepsy, rheumatoid arthritis, and even a few motor vehicle accidents. Toxoplasma gondii is clearly affecting animals and humans in ways we are just beginning to understand, and common sense would suggest we should not be contaminating our environment with the infective oocysts. Perhaps we should use the seals and other sea mammals that are dying on our beaches, many infected with toxoplasmosis, as sentinels. As a past generation used a canary in the coal mine as a sentinel, this generation might use a dead seal on a beach.

Solutions to the toxoplasmosis problem involve four priority actions.

\subsubsection{Decrease the Distribution of Infective T. gondii Oocysts}

The first is to decrease the distribution of the infective T. gondii oocysts in the environment. Since this distribution is being done by infected cats, the decrease can only be accomplished by decreasing the number of infected cats. Owned cats that are exclusively indoor cats are not a problem. They could theoretically become infected with $T$. gondii indoors if they caught a mouse that was infected, but the likelihood of this happening is low. Owned cats that go outdoors are a problem insofar as a significant percentage of them will become infected, most often at the time when they are starting to hunt. For such cats it is essential that the cat litter be cleaned each day, before the feces has dried and the oocysts have become aerosolized, and disposed of in the garbage, not flushed down the toilet. The ultimate solution to having a safe indoor and outdoor cat is the development of an effective vaccine, as will be described below.

The major source of the distribution of $T$. gondii oocysts in the environment are unowned and feral cats. Some of these cats were owned by people at one time but ran away or were left behind when the people moved, but the majority of them were never owned. As previously noted they are estimated to number between 30 and 80 million in the United States. Feral cats are not only responsible for a disproportionate share of the T. gondii oocysts being widely distributed on the land and in the water, they are also responsible for a disproportionate share of feline killings of birds and small mammals such as mice, squirrels, and rabbits. In the United States, it has been estimated that cats kill between 1 and 4 billion birds and between 6 and 22 billion small mammals each year; of this total, two thirds have been attributed to feral cats. Cats are highly effective predators; for example, a single feral cat was shown to have killed 46 out of 52 chickens on 1 farm over 4 nights. In Australia, which is estimated to have 2.7 million owned cats and at least 2 million feral cats, studies have shown that each year the feral cats kill 400 million birds and more than half a billion native mammals. According to one summary of the problem, "Overall, cats have played a major role in the extinction of 27 Australian species since European settlement and are currently threatening the existence of scores more" $[36,37]$.

Attempts to control the feral cat problem using humane methods have been unsuccessful. Most widely used have been programs that trap, neuter, and return 
feral cats to the wild. Multiple careful studies of these "TNR" programs, as they are known, have demonstrated that they don't work. Despite the dedicated work of volunteers who staff these programs, feral cats are difficult to trap, and neutering is expensive. Cats also reproduce faster than TNR programs can keep up with. The average female cat can become pregnant at 6 months of age, have a pregnancy lasting 9 weeks, and give birth to 3-6 kittens. She can then become pregnant again as soon as 4 weeks after giving birth. It is not uncommon for a female cat to have three litters a year. Indoor cats live on average for 12 to 18 years, feral cats much shorter depending on the conditions. Given its fertility, it is quite possible for a single female cat to have more than 1000 descendants by the time she dies [38].

The only effective means that has been found to date for controlling the cat population has been the killing of unwanted and feral cats. In the United States, approximately 1 million cats are euthanized each year in animal shelters overrun by such cats. A realistic solution to the feral cat problem would involve a significant increase in the number of cats euthanized. Given the passionate feelings of animal lovers in general, and cat lovers in particular, this will be difficult to do.

\subsubsection{Research}

A second priority for solving the toxoplasmosis problem is research. As noted above, the development of a vaccine against $T$. gondii is essential. Ideally it should be a vaccine that can be given to kittens to prevent them from ever becoming infected. Such cats would be regarded as safe pets for both children and adults, even if the cats could go outside. Farmers who keep cats in the barn would also vaccinate their kittens so that the animal feed would not become contaminated by infective oocysts. Developing a vaccine for kittens could be done using a live, attenuated strain of the parasite. Such a vaccine has been developed for use in sheep for which toxoplasmosis is a major cause of abortion; it is available in United Kingdom and some other countries but not in the United States or Canada. The development of a vaccine for kittens would not be easy because of the many strains of $T$. gondii and because the parasite is much more complex than a virus. However the recent success of vaccine development against the SARS-CoV-2 virus demonstrated what can be done when such a task is prioritized [39-41].

Unfortunately, in April 2019, animal rights activists made vaccine development more difficult. They lobbied members of congress and convinced them to introduce legislation, called the KITTEN Act, to close the toxoplasmosis research laboratory under the US Department of Agriculture because it was using cats as research subjects. This laboratory, which had been doing research on toxoplasmosis since 1982, was the single most important American research laboratory on this disease and its closure is a major setback for such research [42].

Much research needs to be done on human toxoplasmosis. If it can be proven to be causing even $20 \%$ of cases of new onset psychosis, that would be a huge step forward for psychiatric research since such cases could theoretically be prevented. 
Also needed is additional research on whether $T$. gondii infection has an effect on personality characteristics, as some studies have reported, for individuals who are otherwise asymptomatic. For example, are there groups of Americans who have pronounced personality traits such as suspiciousness, impulsivity, or aggressiveness not because this is their normal personality but rather because they are infected with T. gondii? Related questions that need clarification include possible different effects of infection by a tissue cyst or an oocyst, the different effects of the many strains of the parasite, and the importance of the timing of the initial infection. For example, does an infection in children lead to a different outcome than an infection in adults? Research is also needed on the oocysts themselves. For example, how important is transmission by aerosolized oocysts in causing human disease? How important are the host genetics in determining the outcome of $T$. gondii infections? And how important are coinfections with other infectious agents in determining the outcome? In addition to research on psychosis, research is also needed on the other conditions linked by antibody studies to $T$. gondii-specifically brain cancer, epilepsy, rheumatoid arthritis, and motor vehicle accidents.

\subsubsection{Better Treatments}

The third priority action needed to solve the toxoplasmosis problem is the development of better treatments. The standard treatment for infections with $T$. gondii is pyrimethamine, trade name Daraprim, a drug also used to treat malaria. For treating toxoplasmosis pyrimethamine is usually used in combination with sulfadiazine or another sulfonamide antibiotic. For treating infections anywhere except the brain, this combination is moderately effective for most cases but there are significant side effects. For those who cannot tolerate the drug and those who are allergic to sulfa, a variety of other antiparasitic and antibiotic drugs have been tried but none have been better than pyrimethamine. However when T. gondii infects the brain, it is a different story. The blood-brain barrier blocks many drugs from getting into the brain. Furthermore, in the brain, $T$. gondii forms tissue cysts which are metabolically relatively inactive and therefore do not respond well to treatment by many drugs. The bottom line is that we currently have no effective treatment for infections by T. gondii in the brain [43].

Still another problem is the availability of pyrimethamine. This drug was first approved for use in the United States in 1953. For many years it was marketed by GlaxoSmithKline and sold for $\$ 1.00$ a pill. In 2010 Glaxo sold the American marketing rights of Daraprim to CorePharma, which in 2014 sold them to Impax Laboratories, by which time the price of Daraprim had risen to $\$ 13.50$ a pill. In 2015 Impax sold the rights to Turing Pharmaceuticals, a startup company run by a former hedge fund manager. Turing immediately raised the price of Daraprim to $\$ 750$ a pill, thereby landing itself on the front page of the New York Times as another example of a pharmaceutical company legally but outrageously price gouging. Since that time the availability of pyrimethamine in the United States has been less predictable and, for many, unaffordable [44]. 
Given the need for better drugs to treat psychosis, a few attempts have been made to treat patients with schizophrenia using drugs thought to be effective against T. gondii. Between 2009 and 2014, four such randomly controlled trials were reported. Two trials used antibiotics - azithromycin and trimethoprim-and the other two trials used antiparasitic drugs, artemisinin and artemether. None of the four trials reported significant improvement in the symptoms of individuals with schizophrenia. This was not surprising given the fact that none of the four drugs have good penetration into the brain. In addition, three of the four trials used patients who had been sick for many years. To be effective it is quite possible that an antitoxoplasma drug would have to be given in the earlier stages of the illness [45].

Thus there are many reasons to develop better drugs for the treatment of infections by $T$. gondii. But developing better drugs is expensive and generally only done by the pharmaceutical industry or government. The pharmaceutical industry apparently views the problems caused by human toxoplasmosis as being too small to be economically attractive. In the United States, the National Institutes of Health (NIH) unfortunately also regards human toxoplasmosis as being a low priority. This is demonstrated by the Research, Condition, and Disease Categorization (RCDC) database, a list of 292 diseases and conditions for which NIH provides annual data on how much is being spent for research. Among the 292 diseases and conditions, toxoplasmosis does not even make the list. If better drugs are to be developed for the treatment of toxoplasmosis, they are more likely to be developed in Europe or Asia than in the United States.

One place in which to look for better treatments for toxoplasmosis is the development of better antipsychotics. The chemical structure of many effective antiparasitic drugs is very similar to the structure of antipsychotic drugs. As early as 1891, it was reported that the phenothiazine dye, methylene blue, was effective in suppressing the parasite that causes malaria. Sixty years later the first effective antipsychotic, chlorpromazine, was developed; it is also a phenothiazine. Based on this history, we tested 12 antipsychotic and mood stabilizer drugs to ascertain their effectiveness in inhibiting T. gondii in cell culture. All of the 12 drugs except lithium demonstrated some ability to inhibit $T$. gondii, and two of them-the antipsychotic haloperidol and the mood stabilizer valproate-were even more effective than the antibiotic trimethoprim which is used to treat toxoplasmosis and was used in the study as the comparator drug. Thus it is possible that some of the effectiveness of antipsychotic drugs used to treat psychosis comes from the ability to suppress T. gondii or other infectious agents. And it is also possible that the ultimate solution to the toxoplasmosis treatment problem will come from the development of better antipsychotics [46].

Given the complexity of the $T$. gondii organism and its many strains, it will not be easy to develop better treatments for this parasite, but it can be done. One approach is to chemically modify compounds which are already known to possess some antiprotozoal activity. Recent published examples of this approach include studies on endochin-like quinolones [47]; 4-arylquinoline-2-carboxylate derivatives [48]; and a thiazole derivative of artemisinin [49]. Another approach is to screen large numbers of existing drugs and other chemical compounds to 
ascertain which ones have the best ability to inhibit $T$. gondii in cell culture. This approach has also identified large numbers of potentially useful anti-toxoplasma compounds [50-52].

\subsubsection{Education}

A fourth priority action for solving the toxoplasmosis problem is education. Toxoplasma gondii is virtually unknown in the United States except by women who have been pregnant and educated by their obstetricians. Thus most people have no knowledge of the role of cats and oocysts in spreading the disease. For example, animal lovers living in coastal communities will often make great efforts to save the lives of beached seals or other marine mammals, some of which were beached because they were infected with $T$. gondii. Some of these people will then go home and let their cat out. The cat's feces may then be washed by rainwater into a nearby stream that empties into the bay, thereby infecting more seals that may also beach themselves.

Toxoplasmosis-related problems could be significantly reduced in United States if the public was aware of the following facts.

- Cats that have been kept indoors from birth are safe pets for both children and adults.

- Cats that are allowed to go outside are likely to become infected with T. gondii, most often when they're starting to learn to hunt. In most cases the infected cat will have no symptoms, so the owner will have no way of knowing that the cat is infected.

- Feces from a cat litter box should be removed each day, put in a bag, and put in the garbage. It should never be flushed down the toilet.

- Pregnant women and people who are immunocompromised should never clean the cat litter box.

- Sandboxes and loose soil in children's play areas should always be covered when not in use.

- Gloves should be worn when gardening.

- Meat and seafood should be thoroughly cooked.

- Fruits that cannot be peeled and vegetables should be thoroughly washed before eating.

- Finally, treat cats as cats. Real cats are not like Garfield or Rum Tum Tugger, and they are not small humans with fur. They are a different animal species from humans, and it is possible to show affection for them without kissing them on the face. As noted in Chap. 1, cats are known to carry at least 273 infectious agents, 151 of which are known to be shared with humans. That's a heavy burden for a small animal and perhaps that is what made Grumpy Cat grumpy.

Given what we now know about toxoplasmosis, isn't it time to implement these actions? 


\section{References}

1. Dabritz HA, Conrad PA. Cats and toxoplasmosis: implications for public health. Zoonoses Public Health. 2010;57:34-52.

2. Standard boxcars are 55.5 feet in length and there are 2 feet between the boxcars, thus making a train 3,565 feet or .68 miles in length.

3. Dubey JP. Toxoplasmosis of animals and humans. New York: CRC Press; 2010. p. 36, 38.

4. Dubey JP, et al. Infectivity of low numbers of Toxoplasma gondii oocysts to pigs. J Parasitol. 1996;82:438-43.

5. Yilmaz SM, Hopkins SH. Effects of different conditions on duration of infectivity on Toxoplasma gondii oocysts. J Parasitol. 1972;58:938-9.

6. Frenkel JK, et al. Soil survival of Toxoplasma oocysts in Kansas and Costa Rica. Am J Trop Med Hyg. 1975;24:439-43.

7. Dubey JP. Toxoplasma gondii oocyst survival under defined temperatures. J Parasitol. 1998;84:862-5.

8. Lindsey DS, Dubey JP. Long-term survival of Toxoplasma gondii sporulated oocysts in seawater. J Parasitol. 2009;95:1019-20.

9. Frenkel JK, Dubey JP. Toxoplasma and its prevention in cats and men. J Infect Dis. 1972;126:664-73.

10. de Wit LA, et al. Seasonal and spatial variation in Toxoplasma gondii contamination in soil in urban public spaces in California, United States. Zoonoses Public Health. 2020;67(1):70-8. https://doi.org/10.1111/zph.12656.

11. Dabritz HA, et al. Outdoor fecal deposition by free-roaming cats and attitudes of cat owners and nonowners toward stray pets, wildlife, and water pollution. J Am Vet Med Assoc. 2006;229(1):74-81. https://doi.org/10.2460/javma.229.1.74.

12. Dabritz HA, et al. Detection of Toxoplasma gondii-like oocysts in cat feces and estimates of the environmental oocyst burden. J Am Vet Med Assoc. 2007;231(11):1676-84. https://doi. org/10.2460/javma.231.11.1676.

13. Afonso E, et al. Local meteorological conditions, dynamics of seroconversion to Toxoplasma gondii in cats (Felis catus) and oocyst burden in rural environment. Epidemiol Infect. 2010;138:1105-13.

14. dos Santos TR, et al. Detection of Toxoplasma gondii oocysts in environmental samples from public schools. Vet Parasitol. 2010;171:53-7.

15. Sousa OE, et al. Toxoplasmosis in Panama: A 10 year study. Am J Trop Med Hyg. 1988;38:315-22.

16. Pacheco-Ortega GA, et al. Screening of zoonotic parasites in playground sandboxes of public parks from subtropical Mexico. J Parasitol Res. 2019;2019:7409076. https://doi. org/10.1155/2019/7409076.

17. Lass A, et al. Detection of Toxoplasma gondii oocysts in environmental soil sample using molecular methods. Eur J Clin Microbiol Infect Dis. 2009;28:599-605.

18. Du F, et al. Survey on the contamination of Toxoplasma gondii oocysts in the soil of public parks in Wuhan, China. Vet Parasitol. 2012;184:141-6.

19. Saki J, et al. Detection and genotyping of Toxoplasma gondii isolated from soil in Ahvaz, Southwest of Iran. J Parasitol Dis. 2017;41:202-5.

20. Ajmal A, et al. Detection of Toxoplasma gondii in environmental matrices (water, soil forests and vegetables). Afr J Microbiol Res. 2013;7:1505-11.

21. Cong W, et al. Prevalence, risk factors and genotype distribution of Toxoplasma gondii DNA in soil in China. Ecotoxicol Environ Saf. 2020;189:109999. https://doi.org/10.1016/j. ecoenv.2019.109999.

22. Maleki B, et al. Toxoplasma oocysts in the soil of public places worldwide: a systematic review and metanalysis. Trans R Soc Trop Med Hyg. 2021;115:471-81.

23. Uga S, et al. Defecation habits of cats and dogs and contamination by Toxocara eggs in public park sandpits. Am J Trop Med Hyg. 1996;54:122-6. 
24. Torrey EF, Yolken RH. Toxoplasma oocysts as a public health problem. Trends Parasitol. 2013;29:380-4.

25. Bowie WR, et al. Outbreak of toxoplasmosis associated with municipal drinking water. The BC Toxoplasma investigation team. Lancet (London, England). 1997;350(9072):173-7. https://doi.org/10.1016/s0140-6736(96)11105-3.

26. Shapiro K, et al. Environmental transmission of Toxoplasma gondii: oocysts in water, soil and food. Food and Waterborne Parasitol. 2019;15:e00049. https://doi.org/10.1016/j.fawpar.2019.e00049.

27. Miller MA, et al. An unusual genotype of Toxoplasma gondii is common in California sea otters (Enhydra lutris nereis) and is a cause of mortality. Int J Parasitol. 2004;34(3):275-84. https://doi.org/10.1016/j.ijpara.2003.12.008.

28. Gibson AK, et al. Polyparasitism is associated with increased disease severity in Toxoplasma gondii-infected marine sentinel species. PLoS Negl Trop Dis. 2011;5:e1142. https://doi. org/10.1371/journal.pntd.0001142.

29. Dabritz HA, et al. Outdoor fecal deposition by free-roaming cats and attitudes of cat owners and nonowners toward stray pets, wildlife, and water pollution. J Am Vet Med Assoc. 2006;229:74-80.

30. Jessup DA, et al. Sea otters in a dirty ocean. J Am Vet Med Assoc. 2007;231:1648-52.

31. Iqbal A, et al. Toxoplasma gondii infection in stranded St. Lawrence Estuary beluga Delphinapterus leucas in Quebec, Canada. Dis Aquat Org. 2018;130:165-75.

32. Dubey JP, et al. Recent epidemiologic and clinical importance of Toxoplasma gondii infections in marine mammals: 2009-2020. Vet Parasitol. 2020;288:109296. https://doi.org/10.1016/j. vetpar.2020.109296.

33. Cat Parasite Found in Western Arctic Belugas. CBC News. February 17, 2014. https://www. cbc.ca/news/technology/cat-parasite-found-in-western-arctic-belugas-1.2536234.

34. Petersen DR, et al. Prevalence of activity to Toxoplasma among Alaska natives: relation to exposure to the felidae. J Infect Dis. 1974;130:557-63.

35. Jensen S-K, et al. Prevalence of Toxoplasma gondii antibodies in pinnipeds from Antarctica. Vet Rec. 2012;171:249. https://doi.org/10.1136/vr.100848.

36. Losos JB. Deadly cats down under: science meets the great cat debate. Science. 2019;375:328-9. https://doi.org/10.1126/science.aay2196.

37. Strycker N. To save birds should we kill off cats. National Geographic. 2019. Vol. 236. https:// www.nationalgeographic.com/animals/2019/09/essay-to-save-birds-should-we-kill-off-cats/.

38. Read JL. Among the pigeons: why our cats belong indoors. Mile End: Wakefield Press; 2019. p. 111-30.

39. Li Y, Zhou H. Moving toward improved vaccines for Toxoplasma gondii. Expert Opin Biol Ther. 2018;18:273-80.

40. Singh T, et al. Vaccines for perinatal and congenital infections-how close are we? Front Pediatr. 2020;8:569. https://doi.org/10.3389/fped.2020.

41. Chu K-B, Quan F-S. Advances in Toxoplasma gondii vaccines: current strategies and challenges for vaccine development. Vaccine. 2021;9:413. https://doi.org/10.3390/vaccines9050413.

42. Wadman M. Closure of U.S. Toxoplasma lab draws ire. Science. 2019;364:109.

43. Neville AJ, et al. Clinically available medicines demonstrating anti-Toxoplasma activity. Antimicrob Agents Chemother. 2015;59:7161-9.

44. Pollack A. Once a neglected treatment, now an expensive specialty drug. The New York Times, September 21, 2015.

45. Chorlton SD. Toxoplasma gondii and schizophrenia: a review of published RCTs. Parasitol Res. 2017;116:1793-9.

46. Jones-Brando L, Torrey EF, Yolken R. Drugs used in the treatment of schizophrenia and bipolar disorder inhibit the replication of Toxoplasma gondii. Schizophr Res. 2003;62:237-44.

47. Doggett SJ, et al. Endochin-like quinolones are highly efficacious against acute and latent experimental toxoplasmosis. Proc Natl Acad Sci. 2012;109:15936-41.

48. McNulty J, et al. Synthesis and anti-toxoplasmosis activity of 4-arylquinoline-2-carboxylate derivatives. Org Biomol Chem. 2014;12(2):255-60. 
49. Schultz TL, et al. A thiazole derivative of artemisinin moderately reduces Toxoplasma gondii cyst burden in infected mice. J Parasitol. 2014;100:516-21.

50. Hencken CP, et al. Thiazole, oxadiazole, and carboxamide derivatives of artemisinin are highly selective and potent inhibitors of Toxoplasma gondii. J Med Chem. 2010;53:3594-601.

51. Adeyemi OS, et al. Screening of chemical compound libraries identified new anti-Toxoplasma gondii agents. Parasitol Res. 2018;117:355-63.

52. Murata Y, et al. Identification of compounds that suppress Toxoplasma gondii tachyzoites and bradyzoites. PloS One. 2017. https://doi.org/10.1371/journal.pone.0178203.

Open Access This chapter is licensed under the terms of the Creative Commons AttributionNonCommercial-NoDerivatives 4.0 International License (http://creativecommons.org/licenses/ by-nc-nd/4.0/), which permits any noncommercial use, sharing, distribution and reproduction in any medium or format, as long as you give appropriate credit to the original author(s) and the source, provide a link to the Creative Commons license and indicate if you modified the licensed material. You do not have permission under this license to share adapted material derived from this chapter or parts of it.

The images or other third party material in this chapter are included in the chapter's Creative Commons license, unless indicated otherwise in a credit line to the material. If material is not included in the chapter's Creative Commons license and your intended use is not permitted by statutory regulation or exceeds the permitted use, you will need to obtain permission directly from the copyright holder.

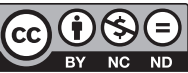

